\title{
Protein kinase A antagonizes Hedgehog signaling by regulating both the activator and repressor forms of Cubitus interruptus
}

\author{
Gelin Wang, Bing Wang, and Jin Jiang ${ }^{1}$ \\ Center for Developmental Biology and Department of Pharmacology, University of Texas Southwestern Medical Center, \\ Dallas, Texas 75235-9133 USA
}

The Hedgehog (Hh) family of secreted proteins controls many aspects of animal development. In Drosophila, Hh transduces its signal via Cubitus interruptus (Ci), a transcription factor present in two forms: a full-length activator and a carboxy-terminally truncated repressor that is derived from the full-length form by proteolytic processing. The proteolytic processing of $\mathrm{Ci}$ is promoted by the activities of protein kinase $\mathrm{A}$ (PKA) and Slimb, whereas it is inhibited by Hh. Here we show that PKA inhibits the activity of the full-length Ci in addition to its role in regulating $\mathrm{Ci}$ proteolysis. Whereas $\mathrm{Ci}$ processing is blocked in both $P K A$ and slimb mutant cells, the accumulated full-length $\mathrm{Ci}$ becomes activated only in PKA but not in slimb mutant cells. Moreover, PKA inhibits an uncleavable activator form of $\mathrm{Ci}$. These observations suggest that PKA regulates the activity of the full-length $\mathrm{Ci}$ independent of its proteolytic processing. We also provide evidence that PKA regulates both the proteolytic processing and transcriptional activity of $\mathrm{Ci}$ by directly phosphorylating $\mathrm{Ci}$. We propose that phosphorylation of $\mathrm{Ci}$ by PKA has two separable roles: (1) It blocks the transcription activity of the full-length activator form of $\mathrm{Ci}$, and (2) it targets $\mathrm{Ci}$ for Slimb-mediated proteolytic processing to generate the truncated form that functions as a repressor.

[Key Words: PKA; Ci; Hedgehog; Slimb; proteolysis; limb development]

Received August 12, 1999; revised version accepted September 21, 1999.

Secreted proteins of the Hedgehog (Hh) family govern cell growth and patterning in a wide variety of developmental processes in both vertebrates and invertebrates (Ingham 1994; Hammerschmidt et al. 1997). Moreover, mutations in components of the Hh signaling pathway have been implicated in many human disorders including cancers (Dean 1996; Goodrich and Scott 1998). In Drosophila, $\mathrm{Hh}$ is required at multiple developmental stages and is responsible for patterning embryonic segments and adult structures such as wings, legs, and eyes (Ingham 1994; Hammerschmidt et al. 1997). In the developing wings, posterior $(\mathrm{P})$ compartment cells express and secrete $\mathrm{Hh}$ proteins that diffuse into the anterior (A) compartment and induce neighboring A compartment cells to express decapentaplegic $(d p p)$, which encodes a member of the transforming growth factor- $\beta /$ bone morphogenetic protein (TGF $\beta / \mathrm{BMP}$ ) family of secreted proteins (Basler and Struhl 1994; Capdevila and Guerrero 1994; Tabata and Kornberg 1994). Dpp then diffuses into both $\mathrm{A}$ and $\mathrm{P}$ compartments and functions as a longrange morphogen to control the growth and patterning of cells in the whole wing (Lecuit et al. 1996; Nellen et al.

${ }^{1}$ Corresponding author.

E-MAIL jiang@utsw.swmed.edu; FAX (214) 648-1960.
1996). Although the long-range organizing influence of Hh is mediated primarily by Dpp, Hh functions as a local morphogen to specify patterns near the A/P compartment boundary by activating other genes including patched (ptc) and engrailed (en) (Strigini and Cohen 1997).

Hh transduces its signal through a receptor complex containing the transmembrane protein Ptc and the serpentine protein Smoothened (Smo) (Alcedo and Noll 1997; Ingham 1998). In the absence of Hh, Ptc inhibits the activity of Smo. Hh directly binds to Ptc and alleviates this repression. Smo then signals to activate Cubitus interruptus (Ci), a member of the Gli family of zinc finger transcription factors (Ruiz i Altaba 1997). In imaginal disc development, $\mathrm{Ci}$ has dual roles that are carried out by two distinct forms of Ci. In A compartment cells distant from the A/P compartment boundary, $\mathrm{Ci}$ is proteolytically cleaved to form a carboxy-terminally truncated form (Ci75) (Aza-Blanc et al. 1997). This form of $\mathrm{Ci}$ functions as a repressor and blocks the expression of $\mathrm{Hh}$ responsive genes such as $d p p$ (Dominguez et al. 1996; Methot and Basler 1999). In A compartment cells near the A/P compartment boundary, Hh signaling prevents the cleavage of $\mathrm{Ci}$ and stimulates the activity of accumulated full-length Ci (Aza-Blanc et al. 1997; 
Methot and Basler 1999). The full-length form of Ci functions as a transcriptional activator and controls the expression of Hh target genes such as ptc and en (Alexandre et al. 1996; Dominguez et al. 1996; Methot and Basler 1999).

Proteolytic processing of $\mathrm{Ci}$ requires the activities of protein kinase A (PKA) and Slimb, an F-box/WD40 repeat-containing protein (Johnson et al. 1995; Jiang and Struhl 1998; Ohlmeyer and Kalderon 1998). Eliminating PKA or Slimb function abrogates Ci proteolysis, so that no truncated Ci repressor forms and full-length $\mathrm{Ci}$ accumulates, and as a consequence, Hh signaling is activated (Jiang and Struhl 1995, 1998; Lepage et al. 1995; Li et al. 1995; Pan and Rubin 1995; Strutt et al. 1995; Theodosiou et al. 1998). Ci contains multiple PKA phosphorylation consensus sites in its carboxy-terminal region, implying that Ci is a PKA substrate (Orenic et al. 1990). In support of this, mutating the PKA phosphorylation sites blocks Ci processing in tissue culture cells, suggesting that phosphorylation of $\mathrm{Ci}$ by PKA targets $\mathrm{Ci}$ for proteolytic processing (Chen et al. 1998). Recent studies suggest that the vertebrate homolog of Slimb, $\beta$ TRCP, specifies the substrates of the so-called SCF (Skp1, Cdc53, and F-box) ubiquitin ligase complex. This complex targets phosphorylated substrates (such as IкB and $\beta$-catenin) for ubiquitination followed by proteasome-mediated proteolysis (Maniatis 1999). By analogy, it has been proposed that Slimb acts downstream of PKA to target phosphorylated Ci for ubiquitin/proteasome-mediated proteolysis (Jiang and Struhl 1998; Maniatis 1999).

Here we provide in vivo evidence that phosphorylation of Ci by PKA not only targets Ci for Slimb-mediated proteolysis but also inhibits the activity of the fulllength activator form of $\mathrm{Ci}$ independent of its processing. We show that although Ci processing is blocked both in PKA and slimb mutant cells, the accumulated fulllength form of $\mathrm{Ci}$ is only activated in PKA but not in slimb mutant cells. Moreover, PKA can inhibit the activity of an uncleavable activator form of Ci. We demonstrate that both the proteolytic processing and the transcriptional activity of the full-length $\mathrm{Ci}$ are regulated by PKA phosphorylation of Ci. Finally, we show that PKA and $\mathrm{Su}(\mathrm{fu})$ act through distinct mechanisms to inhibit the activity of the full-length $\mathrm{Ci}$.

\section{Results}

\section{High levels of constitutive PKA activity do not promote Ci processing in slimb mutant cells}

PKA and Slimb are both required for proteolytic processing of Ci (Jiang and Struhl 1998). To determine the epistatic relationship between PKA and Slimb in regulating Ci processing, we generated slimb mutant clones that express high levels of a constitutively active form of PKA $\left(\mathrm{mC}^{\star}\right.$, Li et al. 1995) and assayed them for Ci processing by immunostaining with a $\mathrm{Ci}$ antibody (2A1), which recognizes the full-length but not the truncated form of $\mathrm{Ci}$ (Motzny and Holmgren 1995; Aza-Blanc et al. 1997). $\mathrm{mC}^{\star}$ was expressed from a $U A S-m C^{*}$ transgene under the control of a Gal4 driver line, MS1096, that expresses high levels of Gal4 almost uniformly in the wing pouch region (Fig. 1C, Capdevila and Guerrero 1994). It has been shown that overexpression of $\mathrm{mC}^{\star}$ at high levels in wing discs can override Hh signaling and block ptc expression (Li et al. 1995). Consistent with this observation, overexpression of $\mathrm{mC}^{\star}$ using the MS1096 Gal4 driver line blocks the accumulation of full-length $\mathrm{Ci}$ as well as the expression of ptc-lacZ induced by Hh (Fig. $1 \mathrm{~B}$, see below), suggesting that high levels of PKA activity act dominantly to promote Ci processing. However, overexpression of $\mathrm{mC}^{\star}$ does not promote Ci processing in the absence of slimb function, as anteriorly situated slimb mutant cells that express MS1096/UAS-mC* accumulate high levels of full-length Ci (Fig. 1D-E). These observations are consistent with Slimb acting either downstream of or parallel to PKA in regulating the proteolytic processing of $\mathrm{Ci}$.

\section{Ci is activated in PKA but not in slimb mutant cells}

Previous genetic studies showed that anteriorly situated PKA and slimb mutant clones induced limb duplication as well as ectopic $d p p$ expression (Jiang and Struhl 1995, 1998; Lepage et al. 1995; Li et al. 1995; Pan and Rubin
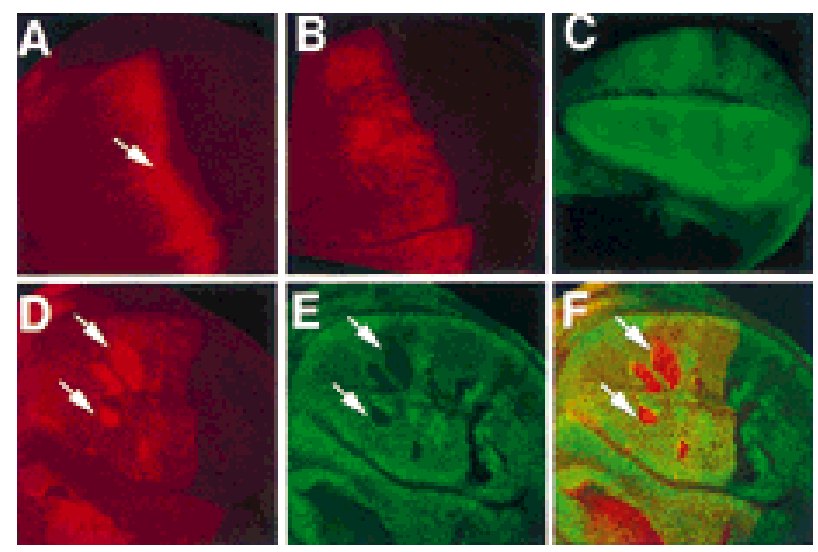

Figure 1. High levels of constitutive PKA activity do not promote Ci processing in slimb mutant cells. (A) A wild-type late third-instar wing disc stained with an anti-Ci antibody (2A1) that recognizes the full-length form of $\mathrm{Ci}$. In this and subsequent figures, all of the wing discs are shown with anterior to the left and ventral up. The arrow indicates the accumulation of full-length $\mathrm{Ci}$ in A compartment cells near the A/P compartment boundary. (B) A late third-instar wing disc expressing $U A S-m C^{\star}$ with MS1096 and stained with 2A1. High levels of constitutive PKA activity block the accumulation of full-length $\mathrm{Ci}$ in A compartment cells near the A/P compartment boundary. (C) A late third-instar wing disc expressing UAS-GFP with MS1096. GFP is expressed almost uniformly in the wing pouch region with slightly higher levels in dorsal compartment cells. The GFP expression reflects the pattern of Gal4 expression driven by the MS1096 Gal4 line. (D-F) A late third-instar wing disc containing slimb clones and expressing UAS- $m C^{*}$ with MS1096 and doubly stained with 2A1 (red in D and F) and a Myc antibody (green in $E$ and $F$ ). slimb mutant cells (marked by the lack of Myc-GFP expression and indicated by the arrows) accumulate high levels of full-length $\mathrm{Ci}$ even though they express high levels of $\mathrm{mC}^{\star}$. 
1995; Theodosiou et al. 1998). However, close examination of phenotypes associated with PKA and slimb mutant clones revealed interesting differences. The supernumerary wings induced by slimb mutant clones are on the average smaller than those induced by $P K A$ mutant clones (Fig. 2, cf. B with C and D). Moreover, the supernumerary wings induced by slimb clones often contain incomplete vein structures (Fig. 2D). In the supernumerary wings, slimb mutant cells contribute to ectopic vein 3 whereas PKA mutant cells contribute to intervein tissues that normally form posteriorly to vein 3 (Fig. 2A-D). Thus the supernumerary wings induced by slimb mu-

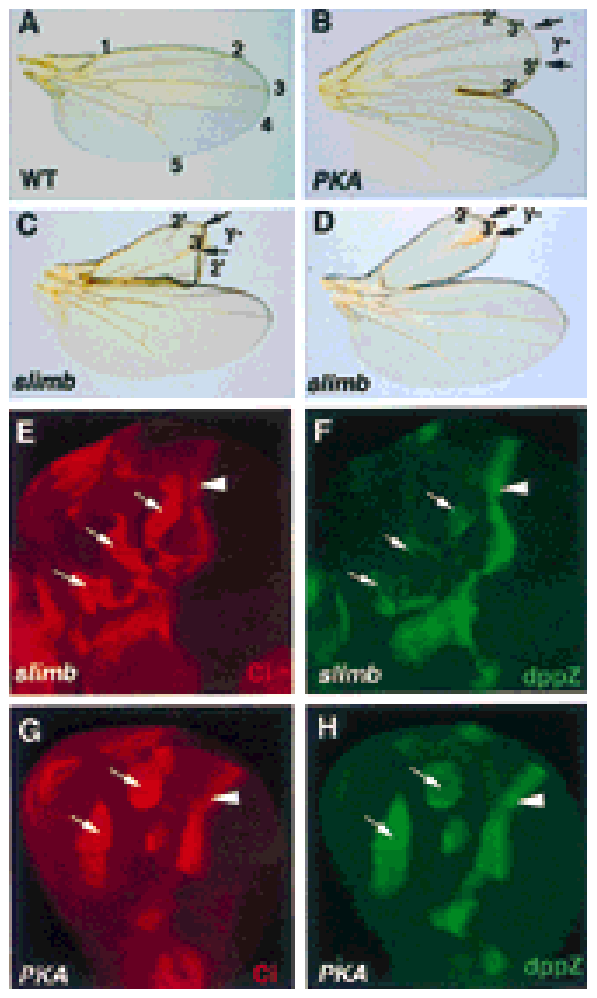

Figure 2. Different phenotypes induced by PKA and slimb mutant clones. (A) A wild-type adult wing with its longitudinal veins indicated by numbers. $(B-D)$ Supernumerary wings organized by a clone of $P K A$ mutant cells $(B)$ or a clone of slimb mutant cells $(C, D)$. Both PKA and slimb mutant cells are marked by $\mathrm{y}^{-}$and are situated between two arrows along the wing margin. In the supernumerary wings, PKA mutant cells form intervein tissues flanked by ectopic vein 3; slimb mutant cells form ectopic vein3. The supernumerary wings organized by slimb clones are smaller than that induced by the PKA clone and contain incomplete veins $(C, D) .(E, F)$ A late third-instar wing disc carrying slimb clones and showing the accumulation of full-length $\mathrm{Ci}(E)$ and $d p p-1 a c Z$ expression $(F)$. Anteriorly situated slimb mutant clones (indicated by the arrows) accumulate high levels of full-length $\mathrm{Ci}$ and express $d p p-l a c Z$ at levels lower than those of the endogenous $d p p-1 a c Z$ at the A/P compartment boundary (arrowhead). $(G, H)$ A late third-instar wing disc carrying $P K A$ mutant clones and showing $\mathrm{Ci}$ accumulation $(G)$ and $d p p-l a c Z$ expression $(H)$. Anteriorly situated $P K A$ mutant clones (arrows) accumulate high levels of full-length $\mathrm{Ci}$ and express $d p p-1 a c Z$ at levels comparable to those of the endogenous $d p p-1 a c Z$ (arrowhead). tant clones resemble more closely those induced by low levels of $d p p$ expression than those induced by ectopic hh expression (Basler and Struhl 1994; Zecca et al. 1995). These observations imply that not all of the outputs of Hh signaling are induced in slimb mutant cells.

To compare slimb and PKA mutant phenotypes molecularly, we examined the expression of $d p p-1 a c Z$ reporter genes in $P K A$ and slimb mutant clones. Although both slimb and PKA mutant clones accumulate fulllength $\mathrm{Ci}$ at comparable levels (Fig. 2E,G), slimb mutant clones ectopically express $d p p-l a c Z$ at levels lower than those of endogenous $d p p-l a c Z$ at the $\mathrm{A} / \mathrm{P}$ compartment boundary (Fig. 2F), whereas PKA mutant cells fully activate $d p p-1 a c Z$ (Fig. $2 \mathrm{H}$ ). The low levels of $d p p-1 a c Z$ expression observed in slimb mutant clones may explain the smaller size of the supernumerary wings induced by slimb mutant clones, as Dpp functions in a concentration-dependent manner to control the growth and patterning of the wings (Zecca et al. 1995; Lecuit et al. 1996; Nellen et al. 1996).

We next examined whether genes regulated by the full-length activator form of $\mathrm{Ci}$, such as ptc and en, are expressed in slimb mutant cells. Anteriorly situated slimb mutant clones in the wing pouch region fail to induce ectopic expression of ptc-lacZ (Fig. 3B), nor do they ectopically express en (data not shown). In contrast, PKA mutant clones situated in similar regions activate ptc-lacZ (Fig. 3A) and express low levels of en (Ohlmeyer and Kalderon 1998). Because ptc and en are regulated by the full-length activator form of $\mathrm{Ci}$, these results suggest that the full-length $\mathrm{Ci}$ that accumulates in slimb mutant cells is transcriptionally silent. The low levels of ectopic $d p p-l a c Z$ expression observed in slimb mutant cells are likely due to derepression of $d p p$, as Ci processing, which generates the repressor form of $\mathrm{Ci}$, is blocked in slimb mutant cells. Although the result shown in Figure 3B was obtained using a hypomorphic slimb allele, $\operatorname{sim} b^{1}$, similar result was obtained with a slimb null allele, $\operatorname{slimb}^{P}$ (Jiang and Struhl 1998; data not shown). This argues against the possibility that the full-length $\mathrm{Ci}$ accumulated in slimb mutant cells is inactive because it is trapped in an SCF complex containing Slimb.

Two possible models might explain the differences between PKA and slimb mutant phenotypes. In addition to its role in regulating $\mathrm{Ci}$ proteolysis, Slimb might play a positive role in transducing $\mathrm{Hh}$ signal. Alternatively, PKA might repress the activity of the full-length activator form of Ci even when Ci processing is blocked. To distinguish between these two possibilities, we examined the activity of $\mathrm{Ci}$ in cells deficient for both Slimb and PKA activities. To generate PKA and slimb double mutant cells, we induced slimb mutant clones in wing discs expressing a mutant PKA regulatory subunit, $\mathrm{R}^{*}$, from a UAS- $R^{*}$ transgene under the control of the MS1096 Gal4 line. Expression of $\mathrm{R}^{*}$ reduces PKA catalytic activity by sequestering the catalytic subunit in the inactive holoenzyme (Li et al. 1995; Ohlmeyer and Kalderon 1997). As shown in Figure 3C, slimb mutant cells expressing $\mathrm{R}^{\star}$ activate $p t c-1 a c Z$ expression, suggesting that slimb mutant cells are competent to activate $p t c-$ 

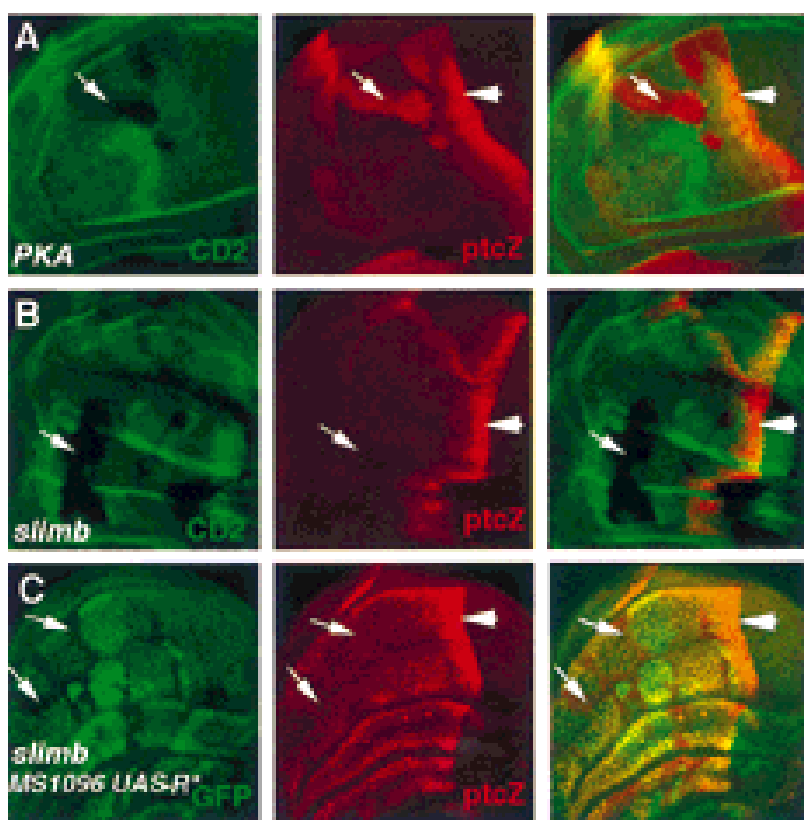

Figure 3. $P K A$ but not slimb mutant cells ectopically express ptc-lacZ. $(A, B)$ Late third-instar wing discs carrying PKA $(A)$ or slimb $(B)$ mutant clones and doubly stained for the marker gene (CD2) expression (green in left and right panels) and ptc-lacZ expression (red in middle and right panels). Both PKA and slimb mutant clones are marked by the lack of CD2 expression. Anteriorly situated PKA mutant cells ectopically express ptc-lacZ (arrow in $A$ ); slimb mutant cells do not (arrow in $B)$. (C) A late third-instar wing disc carrying slimb mutant clones and expressing MS1096/UAS-R* The disc was doubly stained for GFP expression (green in left and right panels) and ptc-lacZ expression (red in middle and right panels). slimb mutant cells are marked by the lack of GFP expression (arrows). Anteriorly situated slimb mutant cells with reduced PKA activity express $p t c-1 a c Z$. The levels of ectopic ptc-lacZ expression are lower than those at the compartment boundary because expressing $U A S-R^{*}$ only partially eliminates PKA activity.

lacZ but are normally blocked by PKA. This observation argues against a positive role for slimb in activating $\mathrm{Ci}$ and suggests an inhibitory role for PKA in keeping the full-length form of $\mathrm{Ci}$ inactive prior to its proteolytic processing.

\section{PKA inhibits an uncleavable activator form of $\mathrm{Ci}$}

Overexpression of a constitutively active form of PKA $\left(\mathrm{mC}^{\star}\right)$ at high levels can override Hh signaling to block Hh-mediated activation of ptc (Li et al. 1995). In agreement with this previous observation, wing discs expressing UAS-mC* under the control of the MS1096 Gal4 driver line showed disruption in ptc-lacZ expression domain at the A/P compartment boundary (Fig. 4B). To obtain further evidence that PKA inhibits the activator form of $\mathrm{Ci}$ independent of its ability to promote Ci processing, we asked whether overexpression of $\mathrm{mC}^{\star}$ could suppress the activity of an uncleavable activator form of $\mathrm{Ci}, \mathrm{Ci}^{\mathrm{U}}$, which contains a small deletion (from amino acid 612 to amino acid 760 ) that removes the cleavage site (Methot and Basler 1999). It has been shown that $\mathrm{Ci}^{\mathrm{U}}$ cannot be processed to generate the repressor form of $\mathrm{Ci}$ but can still mediate Hh-dependent activation of target genes (Methot and Basler 1999). We coexpressed $\mathrm{mC}^{\star}$ and a HA-tagged form of $\mathrm{Ci}^{\mathrm{U}}\left(\mathrm{HACi}^{\mathrm{U}}\right)$ using the MS1096 Gal4 line and determined whether $\mathrm{mC}^{\star}$ could suppress the activity of $\mathrm{HACi}^{\mathrm{U}}$. Wing discs expressing MS1096/ $H A C i^{U}$ ectopically expressed $p t c-l a c Z$ at uniformly high levels in the $\mathrm{P}$ compartment (Fig. 4C), consistent with the previous observation that the activity of $\mathrm{Ci}^{\mathrm{U}}$ depends on Hh signaling (Methot and Basler 1999). In contrast, wing discs coexpressing $\mathrm{HACi}^{\mathrm{U}}$ and $\mathrm{mC}^{\star}$ showed reduction in the levels of ptc-lacZ expression, which is more evident in A compartment cells near the compartment boundary (Fig. 4D). This observation suggests that overexpression of $\mathrm{mC}^{\star}$ can override $\mathrm{Hh}$ signaling to suppress the activity of $\mathrm{Ci}^{\mathrm{U}}$ even though this form of Ci cannot be processed. Taken together, both loss-of-function and gain-of-function studies provide strong evidence that PKA negatively regulates the transcriptional activity of the full-length $\mathrm{Ci}$ independent of $\mathrm{Ci}$ cleavage.

\section{Effects of mutating PKA phosphorylation sites on Ci processing and activity in vivo}

To determine whether the PKA phosphorylation sites are required for $\mathrm{Ci}$ proteolytic processing in vivo, we generated transformants expressing wild-type HACi or
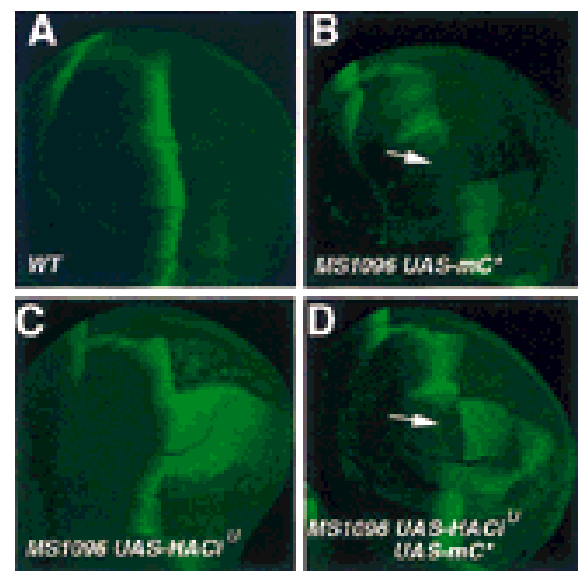

Figure 4. PKA inhibits the activity of an uncleavable activator form of $\mathrm{Ci}$. Wing discs in all panels were stained for $p t c-l a c Z$ expression. (A) A late third-instar wing disc showing the wildtype ptc-lacZ expression. (B) A wing disc expressing a constitutively active form of PKA catalytic subunit $\left(\mathrm{mC}^{\star}\right)$ under the control of the MS1096 Gal4 line. ptc-lacZ expression is suppressed in the wing pouch region (arrow). (C) A wing disc expressing an uncleavable $\mathrm{Ci}\left(\mathrm{HACi}^{\mathrm{U}}\right)$ with $M S 1096$. ptc-lacZ is ectopically expressed in $\mathrm{P}$ but not in A compartment cells away from the compartment boundary. $(D)$ A wing disc expressing $U A S-H A C i^{U}$ and $U A S-m C^{*}$ under the control of the MS1096 Gal4 line. Ectopic ptc-lacZ expression induced by $\mathrm{HACi}^{\mathrm{U}}$ is partially suppressed by coexpression of $\mathrm{mC}^{\star}$. The arrow indicates a gap in the $p t c-l a c Z$ expression domain. The suppression of $p t c-l a c Z$ expression is more evident in A compartment cells near the $\mathrm{A} / \mathrm{P}$ compartment boundary (indicated by the arrow). 
mutant $\mathrm{HACi}^{-3 \mathrm{P}}$ under the control of UAS promoter. $\mathrm{HACi}^{-3 \mathrm{P}}$ has three PKA phosphorylation sites mutated to Ala (S838A, S856A, and S892A). HACi or $\mathrm{HACi}^{-3 \mathrm{P}}$ was expressed in developing wings using the MS1096 Gal4 driver line. Cell extracts were prepared from corresponding wing discs and analyzed by Western blot using an HA-specific antibody. As shown in Figure 5A, HACi was processed to generate the truncated form of $\mathrm{Ci}(\mathrm{Ci} 75)$ whereas $\mathrm{HACi}^{-3 \mathrm{P}}$ was no longer processed and accumulated only as the full-length form, suggesting that PKA phosphorylation at the carboxy-terminal region of $\mathrm{Ci}$ mediates its processing in vivo.

We next investigated whether mutating the PKA phosphorylation sites in $\mathrm{Ci}$ altered its activity. To do this, we expressed HACi or $\mathrm{HACi}^{-3 \mathrm{P}}$ in wing discs and compared their transcription activity by assaying for ptc-lacZ expression. The upstream activating sequence (UAS) transgenes were expressed using a weak Gal4 driver line, tubulin $>C D 2>G V$, which contains a flp-out cassette between a tubulin promoter and a Gal4-VP16 fusion gene
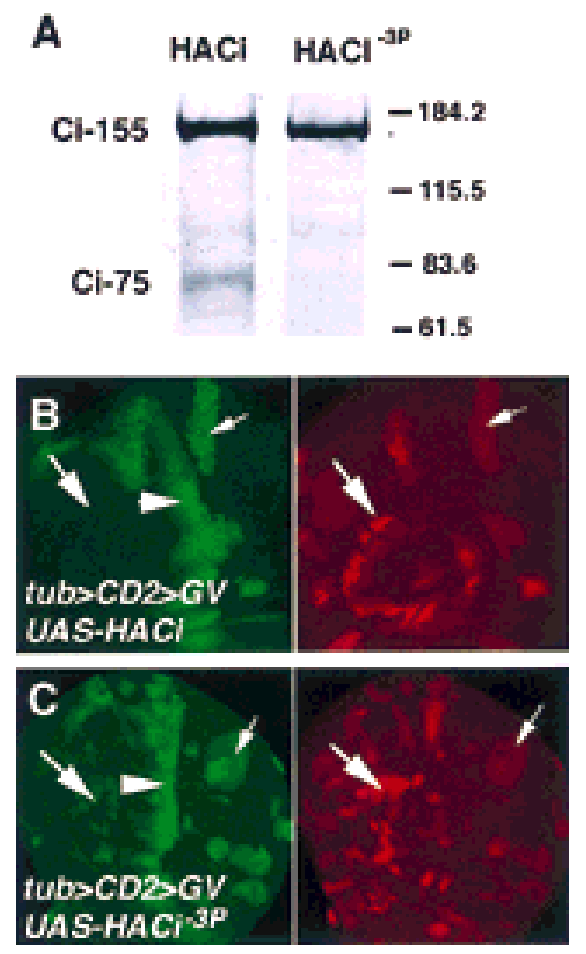

Figure 5. Effects of mutating the PKA phosphorylation sites in $\mathrm{Ci}$ on its processing and activity. (A) Cell extracts from wing discs expressing either HACi or $\mathrm{HACi}^{-3 \mathrm{P}}$ with $\mathrm{MS} 1096$ were probed with an anti-HA antibody. HACi is processed to form the truncated form $(\mathrm{Ci}-75)$ whereas $\mathrm{HACi}^{-3 \mathrm{P}}$ is not. $(B, C)$ Wing discs expressing HACi $(B)$ or $\mathrm{HACi}^{-3 \mathrm{P}}(C)$ under the control of $t u b>C D 2>G V$. The discs were doubly labeled to show $p t c-l a c Z$ expression (left) and HA staining (right). Only P (small arrow) but not A (big arrow) compartment cells that express HACi induce ectopic expression of ptc-lacZ $(B)$. In contrast, both A and $\mathrm{P}$ compartment cells that express $\mathrm{HACi}^{-3 \mathrm{P}}$ ectopically activate $p t c-l a c Z$, although the levels of $p t c-l a c Z$ expression in A compartment cells (big arrow in $C$ ) are lower than those in $\mathrm{P}$ compartment cells (small arrow in $C$ ) or at the compartment boundary (arrowhead).
(Struhl and Adachi 1998). The Gal4-VP16 protein contains a point mutation in the VP16 activating domain to reduce its activity. After removal of the flp-out cassette by Flp-mediated recombination, Ga14-VP16 is expressed under the control of the ubiquitous tubulin promoter. As shown in Figure 5B, clones of cells expressing tub $>G V /$ $U A S-H A C i$ activated $p t c-l a c Z$ when they were situated in the P compartment but failed to do so when they were situated in the A compartment away from the A/P compartment boundary, suggesting that the transcriptional activity of the exogenously expressed wild-type Ci depends on Hh. In contrast, A compartment cells expressing $t u b>G V / H A C i^{-3 P}$ ectopically activated $p t c-l a c Z$, albeit at levels lower than those of endogenous $p t c-l a c Z$ or those induced by $\mathrm{P}$ compartment cells expressing tub $>$ GV $/ U A S-H A C i^{-3 P}$ (Fig. 5C). We examined four independent transformant lines for HACi and five independent lines for $\mathrm{HACi}^{-3 \mathrm{P}}$. None of the HACi lines activated ptc-lacZ in A compartment cells away from the A/P compartment boundary whereas all of the $\mathrm{HACi}^{-3 \mathrm{P}}$ lines induced ectopic ptc-lacZ in A compartment cells although the levels of ectopic ptc-lac $Z$ varied from line to line. In addition, we compared the levels of exogenously expressed $\mathrm{HACi}$ and $\mathrm{HACi}^{-3 \mathrm{P}}$ from multiple transformed lines by staining wing discs with an anti-HA antibody. We found that $\mathrm{HACi}$ and $\mathrm{HACi}^{-3 \mathrm{P}}$ were expressed at comparable levels (Fig. 5B,C), suggesting that the difference in the activity between the wild-type and mutant form of $\mathrm{Ci}$ is due to mutations in the PKA phosphorylation sites rather than difference in the expression levels. Thus, mutating the PKA phosphorylation sites in Ci blocks Ci processing and renders $\mathrm{Ci}$ constitutively active. This suggests that PKA antagonizes Hh signaling by directly phosphorylating $\mathrm{Ci}$. We note that the activity of $\mathrm{HACi}^{-3 \mathrm{P}}$ is still regulated by $\mathrm{Hh}$, as $\mathrm{P}$ compartment cells expressing $\mathrm{HACi}^{-3 \mathrm{P}}$ activated $p t c-1 a c Z$ at levels higher than those induced by A compartment cells expressing the same form of Ci (Fig. 5C). A likely explanation is that other negative regulators of the Hh pathway, such as $\mathrm{Su}(\mathrm{fu})$ and Costal2 (Cos2), may inhibit the activity of $\mathrm{HACi}^{-3 \mathrm{P}}$ in A compartment cells (Robbins et al. 1997; Sisson et al. 1997; Ohlmeyer and Kalderon 1998).

\section{PKA phosphorylation of Ci mediates repression of the activator form of $\mathrm{Ci}$}

Although the above experiments strongly suggest that PKA blocks Hh signaling by directly phosphorylating Ci and targeting it for proteolysis, they do not address whether such phosphorylation also regulates the activity of the full-length activator form of Ci. It is possible that phosphorylation of Ci might only serve to target Ci for processing whereas the observed processing-independent inhibition of $\mathrm{Ci}$ activity by PKA might be indirect. Alternatively, phosphorylation of Ci by PKA may block the activity of the full-length activator form of $\mathrm{Ci}$ independent of $\mathrm{Ci}$ processing. To distinguish between these two possibilities, we investigated whether mutating PKA phosphorylation sites in the uncleavable activator form of $\mathrm{Ci}\left(\mathrm{Ci}^{\mathrm{U}}\right)$ could alter its activity. We mutated the 
same three PKA phosphorylation sites (S838A, S856A, and $\mathrm{S} 892 \mathrm{~A}$ ) in $\mathrm{HACi}^{\mathrm{U}}$ to generate $\mathrm{HACi}^{\mathrm{U}-3 \mathrm{P}}$, and expressed UAS-HACi ${ }^{U}$ or UAS-HACi ${ }^{U-3 P}$ in wings using the MS1096 Gal4 line and assayed for ptc-lacZ expression. Consistent with previous observations, ubiquitously expressing $\mathrm{Ci}^{\mathrm{U}}$ in the wing pouch region induced ectopic ptc-lacZ expression only in the $\mathrm{P}$ compartment but not in the A compartment (Fig. 6A, Methot and Basler 1999). This suggests that the activity of $\mathrm{Ci}^{\mathrm{U}}$ is still inhibited in A compartment cells even though it is not processed and that this inhibition is alleviated by $\mathrm{Hh}$ signaling in P compartment cells. In contrast, expressing $\mathrm{HACi}^{\mathrm{U}-3 \mathrm{P}}$ at comparable levels in the wing pouch region induced ectopic ptc-lacZ expression in both $\mathrm{A}$ and $\mathrm{P}$ compartments (Fig. 6B). We examined four independent transformant lines for $\mathrm{Ci}^{\mathrm{U}}$ and six independent transformant lines for $\mathrm{Ci}^{\mathrm{U}-3 \mathrm{P}}$. None of the $\mathrm{Ci}^{\mathrm{U}}$ lines gave rise to appreciable ectopic $p t c-1 a c Z$ expression in the A compartment whereas all the $\mathrm{Ci}^{\mathrm{U}-3 \mathrm{P}}$ lines ectopically activated ptc-lacZ in both $\mathrm{A}$ and $\mathrm{P}$ compartments. Thus, mutating PKA phosphorylation sites in $\mathrm{HACi}^{\mathrm{U}}$ alleviates the inhibition of HACiU activity in A compartment cells, suggesting that PKA blocks the transcriptional activity of this uncleavable form of $\mathrm{Ci}$ by directly phosphorylating its carboxy-terminal region. We note that the levels of ectopic ptc-lacZ expression induced by $\mathrm{Ci}^{\mathrm{U}-3 \mathrm{P}}$ are lower in A compartment cells than in $\mathrm{P}$ compartment cells, suggesting that $\mathrm{Ci}^{\mathrm{U}-3 \mathrm{P}}$ may still be regulated by other inhibitory components in the Hh pathway.

\section{Inhibition of Ci transcriptional activity by PKA} in slimb $\mathrm{Su}(\mathrm{fu})$ double mutant cells

$\mathrm{Su}(\mathrm{fu})$ has been implicated as an inhibitor of Ci transcriptional activity (Alves et al. 1998; Monnier et al. 1998;

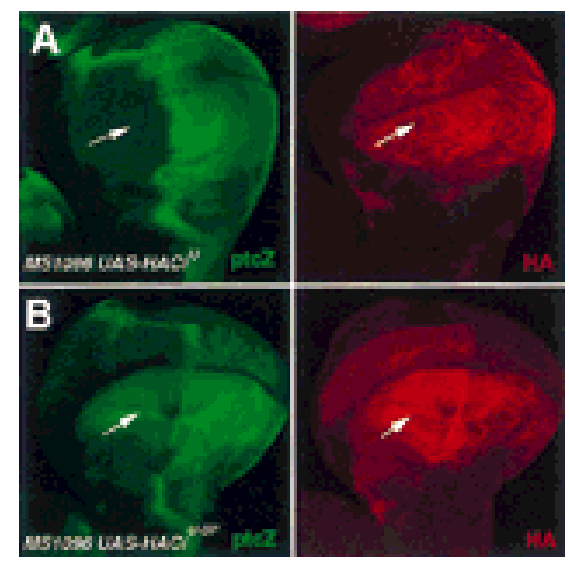

Figure 6. Mutating PKA phosphorylation sites in $\mathrm{Ci}^{\mathrm{U}}$ alters its activity. Wing discs expressing UAS-HACi ${ }^{U}(A)$ or $U A S-$ $H A C i^{U-3 P}(B)$ under the control of the MS1096 Gal4 line were doubly stained for $p t c-l a c Z$ expression (green) and HA expression (red). $\mathrm{HACi}^{\mathrm{U}}$ induces ectopic $p t c-$ lacZ expression only in $\mathrm{P}$ but not in A compartment cells $(A)$. In contrast, $\mathrm{HACi}^{\mathrm{U}-3 \mathrm{P}}$ activates ptc-lac $Z$ in both $\mathrm{A}$ and $\mathrm{P}$ compartment cells $(B)$. Note that the levels of ptc-lacZ in A compartment cells are lower than those in $\mathrm{P}$ compartment cells $(B)$.
Ohlmeyer and Kalderon 1998). To explore the relationship between PKA and $\mathrm{Su}(\mathrm{fu})$ in regulating the fulllength $\mathrm{Ci}$, we examined the expression of ptc-lacZ and en in slimb Su(fu) double mutant and slimb Su(fu) PKA triple mutant cells. We generated slimb Su(fu) PKA triple mutant cells by inducing slimb mutant clones in wing discs homozygous for Su(fu) and expressing UAS$R^{*}$ under the control of the MS1096 Gal4 line. Expressing $U A S-R^{*}$ dramatically reduces but does not completely eliminate PKA activity. Although anteriorly situated slimb singly mutant clones do not induce ectopic ptc-lacZ expression (Fig. 3B), slimb Su(fu) double mutant clones located in similar regions ectopically expressed ptc-lac $Z$, albeit at levels lower than those of the endogenous $p t c-l a c Z$ at the $\mathrm{A} / \mathrm{P}$ compartment boundary (Fig. 7A). This observation suggests that $\mathrm{Su}(\mathrm{fu})$ also contributes to the suppression of Ci activity in slimb mutant cells. However, the relatively low levels of ectopic ptc-lacZ in slimb Su(fu) clones imply that Ci is not fully activated. Consistent with this, slimb Su(fu) double mutant cells failed to activate en (Fig. 7C), which is normally activated only by the highest levels of Hh signaling (Strigini and Cohen 1997). In contrast, anteriorly situated slimb Su(fu) double mutant cells with reduced PKA activity expressed $p t c-l a c Z$ at levels comparable to those at the A/P compartment boundary (Fig. 7B). Moreover, they ectopically expressed en (Fig. 7D). These results suggest that PKA inhibits the activity of the fulllength $\mathrm{Ci}$ independent of $\mathrm{Su}(\mathrm{fu})$ and that PKA and $\mathrm{Su}(\mathrm{fu})$ act in parallel to block the activity of the full-length Ci.

\section{Discussion}

Previous studies in Drosophila and vertebrate model organisms identified PKA as a conserved negative regulator in the Hh signal transduction pathway (for review, see Ingham 1998). Here we investigated the mechanism by which PKA antagonizes Hh signaling activity in vivo. We demonstrate that the transcription factor $\mathrm{Ci}$, which mediates Hh signaling, is a target for PKA. We show that in addition to targeting $\mathrm{Ci}$ for proteolytic processing, which generates the repressor form of $\mathrm{Ci}$, PKA also inhibits the activity of the full-length activator form of $\mathrm{Ci}$. Moreover, we provide evidence that both regulatory events are controlled via phosphorylation of Ci by PKA.

A recent study by Methot and Basler (1999) demonstrated the existence of distinct activator and repressor forms of Ci. These two forms play separable roles in Drosophila limb development by regulating different sets of Hh target genes. In the developing wing, the expression of ptc and en appears to be exclusively controlled by the activator form of $\mathrm{Ci}$ whereas $d p p$ expression is governed by both the activator and repressor forms. Interestingly, preventing $\mathrm{Ci}$ proteolysis with an uncleavable form of $\mathrm{Ci}$ is not sufficient to convert $\mathrm{Ci}$ into a constitutive activator, suggesting that the fulllength activator form of $\mathrm{Ci}$ encounters additional regulatory block(s) that need to be alleviated by Hh signaling (Methot and Basler 1999). Here we provide evidence that PKA activity exerts such a block. Our initial evidence 

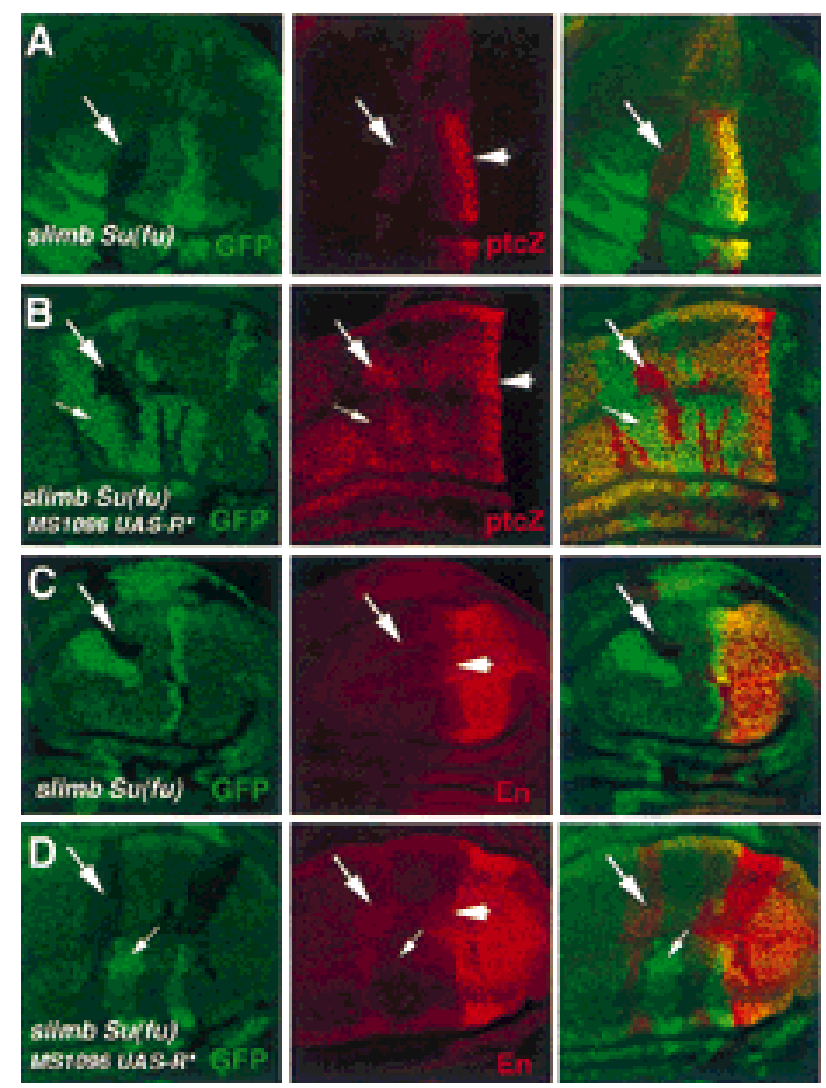

Figure 7. PKA inhibits Ci activity in slimb Su(fu) double mutant cells. Late third-instar wing discs carrying slimb Su(fu) double mutant clones with $(B, D)$ or without $(A, C)$ the expression of a mutant form of PKA regulatory subunit $\left(\mathrm{R}^{\star}\right)$. The wing discs were doubly stained for GFP expression (green) and $p t c-$ lac $Z$ expression (red in $A$ and $B$ ) or En expression (red in $C$ and $D$ ). slimb Su(fu) double mutant clones are marked by the lack of GFP expression (big arrows); the twin clones, which are homozygous for the wild-type slimb and $S u(f u)$ genes, are marked by high levels of GFP expression (small arrows in $B$ and $D$ ). slimb $\mathrm{Su}(\mathrm{fu})$ double mutant cells situated in the A compartment ectopically express low levels of ptc-lacZ (arrow in A) and do not express En (arrow in $C$ ). slimb Su(fu) mutant cells expressing $M S 1096 / U A S-R^{*}$ ectopically activate $p t c-l a c Z$ at high levels (big arrow in $B$ ) as well as En (big arrow in $D$ ). Cells expressing $M S 1096 / U A S-R^{*}$ but homozygous for the wild-type slimb and $\mathrm{Su}(f u)$ genes activate ptc-lacZ at low levels (small arrow in $B$ ) and do not express En (small arrow in $D$ ). Arrowheads in the middle panels indicate the $\mathrm{A} / \mathrm{P}$ compartment boundary.

that PKA regulates the activator form of Ci came from a close examination of $P K A$ and slimb mutant phenotypes. In slimb mutant cells, Ci processing is nearly abolished, and, as a consequence, full-length $\mathrm{Ci}$ accumulates. However, the expression of ptc-lacZ and en is not induced, suggesting that the full-length form of $\mathrm{Ci}$ that accumulates in slimb mutant cells is transcriptionally silent. In contrast, PKA mutant cells express ptc-lac Z and en even though they accumulate full-length $\mathrm{Ci}$ at comparable levels as slimb mutant cells. This suggests that the full-length $\mathrm{Ci}$ that accumulates in PKA mutant cells is transcriptionally active. Furthermore, slimb mutant cells with reduced PKA activity ectopically express ptc-lacZ, arguing that the lack of $\mathrm{Ci}$ activity in slimb mutant cells is due to an inhibitory role of PKA rather than a positive requirement for Slimb in the Hh signaling pathway. Further evidence that PKA regulates the activator form of $\mathrm{Ci}$ independent of its processing came from the gain-of-function studies. We showed that the ectopic expression of $p t c-1 a c Z$ induced by the uncleavable activator form of $\mathrm{Ci}\left(\mathrm{Ci}^{\mathrm{U}}\right)$ was suppressed by overexpression of a constitutively active form of PKA $\left(\mathrm{mC}^{\star}\right)$.

In support of the view that $\mathrm{Ci}$ is a direct target for PKA in regulating $\mathrm{Hh}$ signaling, we found that a modified form of $\mathrm{Ci}$ with three PKA phosphorylation consensus sites mutated was not processed but exhibited constitutive activity when expressed in the developing wings. Although these observations suggest that PKA antagonizes $\mathrm{Hh}$ signaling by directly phosphorylating $\mathrm{Ci}$ and targeting it for proteolysis, they do not to address whether phosphorylation of $\mathrm{Ci}$ by PKA also regulates the activity of the full-length activator form of Ci. The low levels of constitutive activity exhibited by the PKA phosphorylation-deficient form of Ci could be secondary to the lack of $\mathrm{Ci}$ processing, which results in a dramatic increase in the levels of the full-length activator form of $\mathrm{Ci}$, as it has been shown that overexpression of a fulllength wild type form of $\mathrm{Ci}$ can activate $p t c$ expression in wing discs (Alexandre et al. 1996). To define the role of PKA phosphorylation in regulating the activity of the full-length $\mathrm{Ci}$, we took advantage of the uncleavable activator form of $\mathrm{Ci}\left(\mathrm{Ci}^{\mathrm{U}}\right)$ (Methot and Basler 1999). We found that mutating multiple PKA phosphorylation sites in $\mathrm{Ci}^{\mathrm{U}}$ dramatically altered its transcriptional activity and rendered it constitutively active (Fig. 6). This observation suggests that the activity of $\mathrm{Ci}^{\mathrm{U}}$ is normally blocked by PKA phosphorylation even though its processing is impaired. This result provides compelling evidence that PKA phosphorylation of $\mathrm{Ci}$ inhibits the activator form of $\mathrm{Ci}$ independent of its role in promoting $\mathrm{Ci}$ processing. Taken together, our results suggest the following working model for the inhibitory function of PKA in the Hh pathway (Fig. 8). We propose that PKA phosphorylation of $\mathrm{Ci}$ in its carboxy-terminal region has two separable roles: (1) It blocks the activity of the fulllength activator form of $\mathrm{Ci}$, and (2) it targets the fulllength Ci for Slimb-mediated proteolysis to generate the truncated repressor form of $\mathrm{Ci}$. Such a dual regulation ensures that only the repressor form of $\mathrm{Ci}$ is active in the absence of any Hh signaling. This model accounts for the difference between PKA and slimb mutant phenotypes. In slimb mutant cells, $\mathrm{Ci}$ is not processed to the repressor form but accumulates in an inactive phosphorylated form, and, as a consequence, $d p p$ is derepressed at low levels but ptc and en are not activated. In PKA mutant cells, however, Ci accumulates in an unphosphorylated or hypophosphorylated active form, and, as a consequence, ptc and en are activated.

How phosphorylation of $\mathrm{Ci}$ regulates its activity and proteolytic processing remained to be explored. It has been proposed that $\mathrm{Su}(\mathrm{fu})$ attenuates Hh signaling activ- 


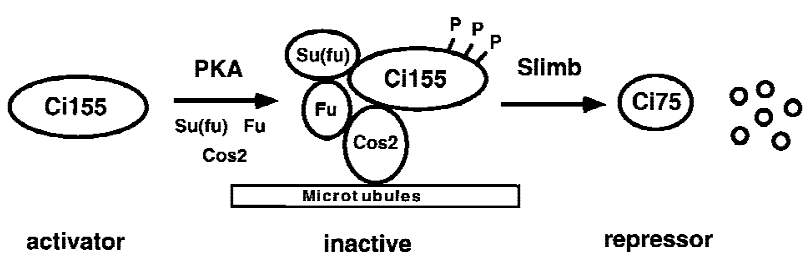

Figure 8. Model for dual regulation of Ci by PKA. Phosphorylation of $\mathrm{Ci}$ in its carboxy-terminal region by PKA keeps the full-length $\mathrm{Ci}$ (Ci155) in an inactive form and targets it for Slimb-mediated proteolytic processing to generate the truncated repressor form (Ci75). Su(fu) and Cos2 also negatively regulate $\mathrm{Ci}$ by forming a complex with $\mathrm{Ci}$ (see text for details).

ity by blocking a maturation step that converts $\mathrm{Ci}$ into a short-lived nuclear transcriptional activator (Ohlmeyer and Kalderon 1998). Our analyses of slimb Su(fu) double mutant and slimb Su(fu) PKA triple mutant phenotypes suggest that inhibition of $\mathrm{Ci}$ activity by PKA is independent of $\mathrm{Su}(\mathrm{fu})$. When Ci processing is blocked, removing $\mathrm{Su}(\mathrm{fu})$ only partially stimulates the activity of the fulllength Ci whereas simultaneously removing $\mathrm{Su}(\mathrm{fu})$ function and reducing PKA activity leads to virtually full activation of Ci (Fig. 7). These observations suggest that PKA and $\mathrm{Su}(\mathrm{fu})$ act in parallel through independent mechanisms to regulate the activity of the full-length $\mathrm{Ci}$. In slimb Su(fu) double mutant cells, the majority of unprocessed full-length $\mathrm{Ci}$ appears to be transformed into a labile nuclear form (Ohlmeyer and Kalderon 1998), and yet the activity of this nuclear form of $\mathrm{Ci}$ seems to be inhibited by PKA (Fig. 7). This implies that PKA might inhibit $\mathrm{Ci}$ at a step after it enters the nucleus. For example, phosphorylation of Ci by PKA might prevent the formation of an active Ci transcription complex or might attenuate its ability to bind DNA. Another possible mechanism by which PKA exerts its influence on $\mathrm{Ci}$ is to regulate its nuclear trafficking. It has been shown recently that $\mathrm{Hh}$ signaling increases the nuclear import of full-length Ci (Chen et al. 1999). As PKA and Hh act antagonistically, it is possible that PKA phosphorylation of Ci might tether the full-length $\mathrm{Ci}$ in the cytoplasm in the absence of Hh signaling.

$\mathrm{Su}(\mathrm{fu}), \mathrm{Cos} 2$, and the Ser/Thr kinase Fu form a multiprotein complex with $\mathrm{Ci}$ and the complex associates with microtubules in a manner regulated by Hh (Robbins et al. 1997; Sisson et al. 1997; Monnier et al. 1998). It has been proposed that the assembly of the microtubule-associated Ci complex is critical for inhibiting $\mathrm{Ci}$ activity, possibly by tethering $\mathrm{Ci}$ in the cytoplasm /Chen et al. 1999). The relationship between PKA phosphorylation and the formation of Ci complex is not known. It is not clear whether they are two independent processes or whether one step might regulate the other. The nearly identical phenotypes caused by loss of PKA or Cos2 function in limb development suggest that these two regulatory events might be intimately related. For example, Cos2 might target $\mathrm{Ci}$ for efficient phosphorylation by PKA, allowing PKA to exert its negative regulation on Ci. Alternatively, phosphorylation of Ci by PKA might regulate the complex formation, allowing Cos2 to exert its influence on Ci. Further genetic and biochemical studies are required to resolve this important issue.

It has been proposed that phosphorylation of $\mathrm{Ci}$ by PKA allows Slimb to bind Ci and target it for ubiquitin/ proteasome-mediated proteolysis (Jiang and Struhl 1998). The epistatic relationship between PKA and Slimb defined by this study is consistent with this hypothesis (Fig. 1). Moreover, it has been shown recently that proteasome is involved in $\mathrm{Ci}$ proteolytic processing (Chen et al. 1999). However, no evidence has been obtained to indicate that $\mathrm{Ci}$ is ubiquitinated /Chen et al. 1999). It is possible that the polyubiquitin chains added to Ci might be unstable and thus might escape detection. Alternatively, the proteolytic processing of Ci might not be directly mediated by ubiquitination, and Slimb might regulate $\mathrm{Ci}$ processing indirectly. For example, the $\mathrm{SCF}^{\text {Slimb }}$ might promote the ubiquitination and degradation of an inhibitor of a protease that cleaves Ci.

Another important question that remains largely unanswered is how Hh antagonizes PKA. The structural similarity between Smo and G protein-coupled seventransmembrane receptors suggests that $\mathrm{Hh}$ signaling might antagonize PKA by down-regulating its cAMP dependent kinase activity (Alcedo et al. 1996). However, the observations that a constitutively active cAMP independent form of PKA $\left(\mathrm{mC}^{\star}\right)$ can rescue PKA mutant phenotypes without perturbing normal $\mathrm{Hh}$ signaling both in embryos and in imaginal discs strongly argue against this possibility (Jiang and Struhl 1995; Li et al. 1995; Ohlmeyer and Kalderon 1997). The finding that high but not low levels of $\mathrm{mC}^{\star}$ are able to override $\mathrm{Hh}$ signaling is more consistent with a model in which $\mathrm{Hh}$ and PKA act competitively and antagonistically on Ci (Jiang and Struhl 1995; Li et al. 1995). For example, Hh may activate a phosphatase that removes the phosphates added to Ci by PKA. In support of this view, pharmacological evidence suggests that Hh stimulates target gene expression via a PP2A-like phosphatase in tissue culture cells (Krishnan et al. 1997; Chen et al. 1999). However, there is no genetic evidence for the involvement of a phosphatase in the Hh pathway.

In vertebrates, Hh signaling is mediated by three members of the Gli family of transcription factors: Gli1, Gli2, and Gli3 (Ruiz i Altaba 1997). Like Ci, all three Gli proteins contain multiple PKA phosphorylation consensus sites at conserved positions, so they are likely to be direct targets for PKA regulation in the vertebrate $\mathrm{Hh}$ signaling pathway. Among the three Gli proteins, Gli3 is both structurally and functionally related to $\mathrm{Ci}$. Gli3 has been implicated to have both activator and repressor function depending on the developmental contexts (Buscher et al. 1997; Motoyama et al. 1998; Ruiz i Altaba 1998, 1999; Dai et al. 1999). Moreover, PKA appears to promote Gli3 processing to generate a putative repressor form (Dai et al. 1999; Ruiz i Altaba 1999). Thus, the mechanism by which PKA targets Ci for Slimb-mediated processing may well be conserved from invertebrates to vertebrates. Gli1 and Gli2 appear to function mainly as positive regulators in the vertebrate $\mathrm{Hh}$ signaling pathway (Lee et al. 1997; Ding et al. 1998; Matise et al. 1998). 
Unlike Gli3 and Ci, Gli1 and Gli2 do not undergo PKAdependent processing (Dai et al. 1999; Ruiz i Altaba 1999|, however, their activities are likely to be regulated by PKA. For example, it has been shown that overexpression of a constitutive active form of PKA represses the transcriptional activity of Glil in mammalian culture cells (Ruiz i Altaba 1999). Thus, whereas the mechanism by which PKA regulates Ci processing may only apply to Gli3, the processing-independent inhibitory mechanism defined by this study may well apply to all three Gli proteins and is likely to be a more general mechanism by which PKA negatively regulates Hh signaling.

\section{Material and methods}

\section{Transgenes and mutations}

To construct UAS-HACi, a double HA-epitope tag was added to the amino terminus of $\mathrm{Ci}$ at the MluI site, and the HA-tagged $\mathrm{Ci}$ was inserted between the $K p n I$ and $X b a I$ sites in the pUAST vector (Brand and Perrimon 1993). UAS-HACi ${ }^{-3 P}$ was derived from UAS-HACi with three PKA phosphorylation consensus sites mutated (S838A, S856A, and S892A) by PCR-based sitedirected mutagenesis. $U A S-H A C i^{-3 P}$ was derived from $U A S-$ $H A C i^{U}$ (Methot and Basler 1999), with the same three PKA sites mutated as for $H A C i^{-3 P}$. UAS-R* and $U A S-m C^{*}$ were described in $\mathrm{Li}$ et al. (1995). Reporter genes used for this study were $d p p-$ lacZ (Blackman et al. 1991) and ptc-lacZ (Chen and Struhl 1997); Gal4 lines were MS1096 (Capidevila and Guerrero 1994); tub $>C D 2>G V$ (generated by Konrad Basler, as cited in Struhl and Adachi 1998); marker genes were hsp-CD2 (Jiang and Struhl 1995) and hsp-myc-GFP (Jiang and Struhl 1998). slimb1 is a hypomorphic allele that is specific for the Hh pathway (Jiang and Struhl 1998), $D C 0^{E 95}$ is an apparently null allele of the DCO gene that encodes the catalytic subunit of PKA (Jiang and Struhl 1998), and $\mathrm{Su}(\mathrm{fu})^{L P}$ is a null allele (Preat 1992).

\section{Generating clones of marked cells}

Clones of mutant cells were generated by FLP/FRT-mediated mitotic recombination (Xu and Rubin 1993). Genotypes for generating clones are as follows: (1) slimb mutant clones: y hspflp.1/y or Y; FRT82B slimb1/FRT82B hsp-CD2, $\mathrm{y}^{+}$(or hsp-my$c-G F P, w^{+}$) with or without $d p p-l a c Z$ or $p t c-l a c Z$ on the second chromosome. (2) PKA mutant clones: y hsp-flp.1/y or $Y$; $D C 0^{E 95}$ stc FRT39, $\mathrm{w}^{+} / \mathrm{hsp}-C D 2, \mathrm{y}+\mathrm{FRT39}, \mathrm{w}^{+}$with or without dpp-lacZ or ptc-lacZ on the third chromosome. (3) slimb Su(fu) mutant clones: y hsp-flp.1/y or Y; FRT82B Su(fu) ${ }^{L P}$ slimb1/ FRT82B, hsp-myc-GFP, $w^{+}$. (4) slimb mutant clones expressing $\mathrm{mC}^{*}$ yw MS1096 hsp-flp/y or Y; UAS-mC*/ptc-lacZ; FRT82B slimb1/FRT82B, hsp-myc-GFP, $\mathbf{w}^{+}$. (5) slimb mutant clones expressing $\mathrm{R}^{\star}$ : yw MS1096 hsp-flp/y or Y; UAS-R*/ptc-lacZ; FRT82B slimb1/FRT82B hsp-myc-GFP, $w^{+}$. (6) slimb Sulfu) mutant clones expressing $\mathrm{R}^{\star}$ : yw MS1096 hsp-flp/y or Y; UAS-R* / ptc-lacZ; FRT82B Su(fu) ${ }^{L P}$ slimb1/FRT82B, hsp-myc-GFP, $w^{+}$.

\section{Imaginal disc staining and Western blot analysis}

Standard protocols for immunofluorescence staining and Western blot analysis of imaginal discs were used (Jiang and Struhl 1998). Primary antibodies used for this study are: monoclonal rat anti-Ci antibody, 2A1(Motzny and Holmgren 1995); mouse anti-HA antibody (Santa Cruz); rabbit anti-ßgal (Cappel); mouse anti-Myc (Santa Cruz); monoclonal mouse anti-CD2, OX34 (Seretec); monoclonal mouse anti-En (DSHB, University of Iowa).

\section{Acknowledgments}

We thank Drs. Dan Kalderon, Konrad Basler, and Gary Struhl for providing plasmids and fly stocks. We are grateful to Drs. James Chen, Jon Graff, Mark Henkemeyer, and D.J. Pan for critically reading the manuscript. This work was initiated in Gary Struhl's laboratory. J.J. thanks Dr. Gary Struhl for his generous support and Atsuko Adachi for injecting some of the constructs used in this study. J.J. is a Searle Scholar supported by the Chicago Community Trust, a Eugene McDermott Endowed Scholar in Biomedical Research, and a Leukemia Society Special Fellow.

The publication costs of this article were defrayed in part by payment of page charges. This article must therefore be hereby marked "advertisement" in accordance with 18 USC section 1734 solely to indicate this fact.

\section{References}

Alcedo, J. and M. Noll. 1997. Hedgehog and its Patched-Smoothened receptor complex: a Novel signalling mechanism at the cell surface. Biol. Cenm. 378: 583-590.

Alcedo, J., M. Ayzenzon, T. Von Ohlen, M. Noll, and J.E. Hooper. 1996. The Drosophila smoothened gene encodes a seven-pass membrane protein, a putative receptor for the Hedgehog signal. Cell 86: 231-232.

Alexandre, C., A. Jacinto, and P.W. Ingham. 1996. Transcriptional activation of Hedgehog target genes in Drosophila is mediated directly by the Cubitus interruptus protein, a member of the GLI family of the zinc finger DNA-binding proteins. Genes \& Dev. 10: 2003-2013.

Alves, G., B. Limbourg-Bouchon, H. Tricoire, J. Brissard-Zahraoui, C. Lamour-Isnard, and D. Busson. 1998. Modulation of Hedgehog target gene expression by the Fused serine-threonine kinase in wing imaginal discs. Mech. Dev. 78: 17-31.

Aza-Blanc, P., F. Ramirez-Weber, M. Laget, C. Schwartz, and T. Kornberg. 1997. Proteolysis that is inhibited by Hedgehog targets Cubitus interruptus protein to the nucleus and converts it to a repressor. Cell 89: 1043-1053.

Basler, K. and G. Struhl. 1994. Compartment boundaries and the control of Drosophila limb pattern by hedgehog protein. $\mathrm{Na}$ ture 368: 208-214.

Blackman, R.K., M. Sanicola, L.A. Raftery, T. Gillevet, and W.M. Gelbart. 1991. An extensive 3' cis-regulatory region directs the imaginal disk expression of decapentaplegic, a member of the TGF-beta family in Drosophila. Development 111: 657-666.

Brand, A.H. and N. Perrimon. 1993. Targeted gene expression as a means of altering cell fates and generating dominant phenotypes. Development 118: 401-415.

Buscher, D., B. Bosse, J. Heymer, and U. Ruther. 1997. Evidence for genetic control of Sonic hedgehog by Gli3 in mouse limb development. Mech. Dev. 62: 175-182.

Capdevila, J. and I. Guerrero. 1994. Targeted expression of the signalling molecule decapentaplegic induces pattern duplications and growth alterations in Drosophila wings. EMBO J. 13: 4459-4468.

Chen, Y. and S. Struhl. 1996. Dual roles for patched in sequestering and transducing hedgehog. Cell 87: 553-563.

Chen, C.H., D.P. von Kessler, W. Park, B. Wang, Y. Ma, and P.A. Beachy. 1999. Nuclear trafficking of Cubitus interruptus in the transcriptional regulation of Hedgehog target gene expression. Cell 98: 305-316.

Chen, Y., N. Gallaher, R.H. Goodman, and S.M. Smolik. 1998 Protein kinase A directly regulates the activity and proteolysis of cubitus interruptus. Proc. Natl. Acad. Sci. 95: 23492354 . 
Dai, P., H. Akimaru, T. Tanaka, T. Maekawa, M. Nakafuku, and S. Ishii. 1999. Sonic Hedgehog-induced activation of the Gli1 promoter is mediated by GLI3. J. Biol. Chem. 274: 8143 8152.

Dean, M. 1996. Polarity, proliferation and the hedgehog pathway. Nat. Genet. 14: 245-247.

Ding, Q., J. Motoyama, S. Gasca, R. Mo, H. Sasaki, J. Rossant, and C.C. Hui. 1998. Diminished Sonic hedgehog signaling and lack of floor plate differentiation in Gli2 mutant mice. Development 125: 2533-2543.

Dominguez, M., M. Brunner, E. Hafen, and K. Basler. 1996. Sending and receiving the Hedgehog signal: Control by the Drosophila Gli protein Cubitus interruptus. Science 272: 1621-1625.

Goodrich, L.V. and M.P. Scott. 1998. Hedgehog and patched in neural development and disease. Neuron 21: 1243-1257.

Hammerschmidt, M., A. Brook, and A. McMahon. 1997. The world according to Hedgehog. Trends Genet. 13: 14-21.

Ingham, P.W. 1994. Hedgehog points the way. Curr. Biol. 4: 347-350.

- 1998. Transducing Hedgehog: The story so far. EMBO J. 17: 3505-3511.

Jiang, J. and G. Struhl. 1995. Protein kinase A and Hedgehog signalling in Drosophila limb development. Cell 80: 563 572.

- 1998. Regulation of the Hedgehog and Wingless signalling pathways by the F-box/WD40-repeat protein Slimb. $\mathrm{Na}$ ture 391: 493-496.

Johnson, R.L., J.K. Grenier, and M.P. Scott. 1995. patched overexpression alters wing disc size and pattern: Transcriptional and post-transcriptional effects on hedgehog targets. Development 121: 4161-4170.

Krishnan, V., F.A. Pereira, Y. Qiu, C.H. Chen, P.A. Beachy, S.Y. Tsai, and M.J. Tsai. 1997. Mediation of Sonic hedgehog-induced expression of COUP-TFII by a protein phosphatase. Science 278: 1947-1950.

Lecuit, T., W.J. Brook, M. Ng, M. Callega, H. Sun, and S.M. Cohen. 1996. Two distinct mechanisms for long-range patterning by Decapentaplegic in the Drosophila wing. Nature 381: 387-393.

Lee, J., K.A. Platt, P. Censullo, and A. Ruiz i Altaba. 1997. Gli1 is a target of Sonic hedgehog that induces ventral neural tube development. Development 124: 2537-2552.

Lepage, T., S.M. Cohen, F.J. Diaz-Benjumea, and S.M. Parkhurst. 1995. Signal transduction by cAMP-dependent protein kinase A in Drosophila limb patterning. Nature 373: 711-715.

Li, W., J.T. Ohlmeyer, M.E. Lane, and D. Kalderon. 1995. Function of protein kinase $\mathrm{A}$ in hedghehog signal transduction and Drosophila imaginal disc development. Cell 80: 553-562.

Maniatis, T. 1999. A ubiquitin ligase complex essential for the NF-кB, Wnt/Wingless, and Hedgehog signaling pathways. Genes \& Dev. 13: 505-510.

Matise, M.P., D.J. Epstein, H.L. Park, K.A. Platt, and A.L. Joyner. 1998. Gli2 is required for induction of floor plate and adjacent cells, but not most ventral neurons in the mouse central nervous system. Development 125: 2759-2770.

Methot, N. and K. Basler. 1999. Hedgehog controls limb development by regulating the activities of distinct transcriptional activator and repressor forms of Cubitus interruptus. Cell 96: 819-831.

Monnier, V., F. Dussillol, G. Alves, C. Lamour-Isnard, and A. Plessis. 1998. Suppressor of fused links fused and Cubitus interruptus on the hedgehog signalling pathway. Curr. Biol. 8: $583-586$.

Motoyama, J., J. Liu, R. Mo, Q. Ding, M. Post, and C.C. Hui.
1998. Essential function of Gli2 and Gli3 in the formation of lung, trachea and oesophagus. Nat. Genet. 20: 54-57.

Motzny, C.K. and R. Holmgren. 1995. The Drosophila cubitus interruptus protein and its role in the wingless and hedgehog signal transduction pathways. Mech. Dev. 52: 137-150.

Nellen, D., R. Burke, G. Struhl, and K. Basler. 1996. Direct and long-range action of a DPP morphogen gradient. Cell 85: 357-368.

Ohlmeyer, J.T. and D. Kalderon. 1997. Dual pathways for induction of wingless expression by protein kinase A and Hedgehog in Drosophila embryos. Genes \& Dev. 11: 22502258.

- 1998. Hedgehog stimulates maturation of Cubitus interruptus into a labile transcriptional activator. Nature 396: 749-753.

Orenic, T.V., D.C. Slusarski, K.L. Kroll, and R.A. Holmgren. 1990. Cloning and characterization of the segment polarity gene cubitus interruptus Dominant of Drosophila. Genes \& Dev. 4: 1053-1067.

Pan, D. and G.M. Rubin. 1995. cAMP-dependent protein kinase and hedgehog act antagonistically in regulating decapentaplegic transcription in Drosophila imaginal discs. Cell 80: $543-552$.

Preat, T. 1992. Characterization of Suppressor of fused, a complete suppressor of the fused segment polarity gene of Drosophila melanogaster. Genetics 132: 725-736.

Robbins, D.J., K.E. Nybakken, R. Kobayashi, J.C. Sisson, J.M. Bishop, and P.P. Therond. 1997. Hedgehog elicits signal transduction by means of a large complex containing the kinesin-related protein costal2. Cell 90: 225-234.

Ruiz i Altaba, A. 1997. Catching a Gli-mpse of Hedgehog. Cell 90: 193-196.

-1998. Combinatorial Gli gene function in floor plate and neuronal inductions by Sonic hedgehog. Development 125: 2203-2212.

1999. Gli proteins encode context-dependent positive and negative functions: Implications for development and disease. Development 126: 3205-3216.

Sisson, J.C., K.S. Ho, K. Suyama, and M.P. Scott. 1997. Costal2, a novel kinesin-related protein in the Hedgehog signaling pathway. Cell 90: 235-245.

Strigini, M. and S.M. Cohen. 1997. A Hedgehog activity gradient contributes to AP axial patterning of the Drosophila wing. Development 124: 4697-4705.

Struhl, G. and A. Adachi. 1998. Nuclear access and action of notch in vivo. Cell 93: 649-660.

Struhl, G. and K. Basler. 1993. Organizing activity of wingless protein in Drosophila. Cell 72: 527-540.

Strutt, D.I., V. Wiersdorff, and M. Mlodzik. 1995. Regulation of furrow progression in the Drosophila eye by cAMP-dependent protein kinase A. Nature 373: 705-709.

Tabata, T. and T.B. Kornberg. 1994. Hedgehog is a signaling protein with a key role in patterning Drosophila imaginal discs. Cell 76: 89-102.

Theodosiou, N.A., S. Zhang, W.Y. Wang, and T. Xu. 1998. slimb coordinates wg and dpp expression in the dorsal-ventral and anterior-posterior axes during limb development. Development 125: 3411-3416.

$\mathrm{Xu}, \mathrm{T}$. and G.M. Rubin. 1993. Analysis of genetic mosaics in developing and adult Drosophila tissues. Development 117: 1223-1237.

Zecca, M., K. Basler, and G. Struhl. 1995. Sequential organizing activities of engrailed, hedgehog and decapentaplegic in the Drosophila wing. Development 121: 2265-2278. 


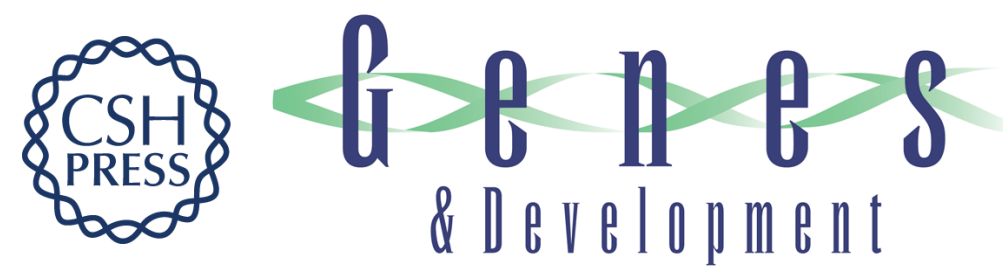

\section{Protein kinase A antagonizes Hedgehog signaling by regulating both the activator and repressor forms of Cubitus interruptus}

Gelin Wang, Bing Wang and Jin Jiang

Genes Dev. 1999, 13:

References This article cites 52 articles, 22 of which can be accessed free at:

http://genesdev.cshlp.org/content/13/21/2828.full.html\#ref-list-1

License

Email Alerting Receive free email alerts when new articles cite this article - sign up in the box at the top Service right corner of the article or click here.

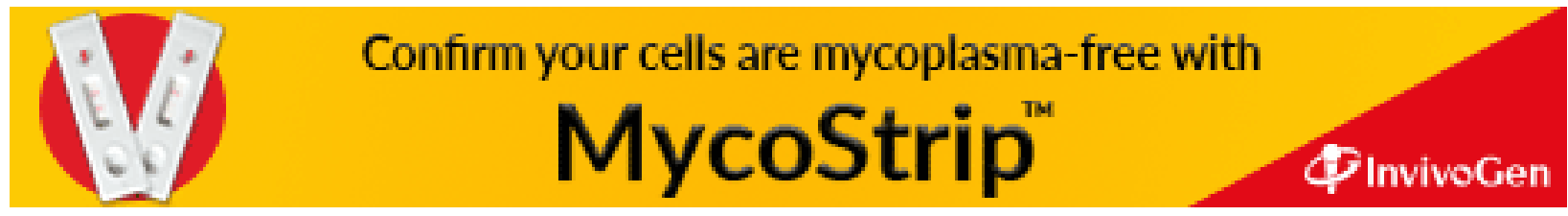

